\title{
Análisis del liderazgo inclusivo en centros educativos de Primaria y Secundaria de Granada
}

\author{
Rubén Moreno-Arrebola. Universidad de Granada \\ María José León Guerrero. Universidad de Granada \\ Recepción: 3 de abril de 2017 | Aceptado: 30 de junio de 2017 \\ Correspondencia: Rubén Moreno | Correo-e: rubenmorenoarrebola@correo.ugr.es
}

(iD 0000-0002-1270-393X

Citar: Moreno, R. y León, MJ. (2017). Análisis del liderazgo inclusivo en centros educativos de Primaria y Secundaria de Granada. ReiDoCrea, 6, 211-220.

\begin{abstract}
Resumen: Una sociedad cada vez más heterogénea, hace necesario el fomento de procesos de inclusión social y educativa. Conociendo que los directivos de los colegios son piezas clave como promotores del cambio y vía privilegiada de mejora, toman sentido los estudios del liderazgo inclusivo. Objetivo: este estudio plantea analizar el liderazgo inclusivo en centros educativos públicos y privados de Granada capital. Método: dicha investigación se llevó a cabo en el curso académico 2015/2016. La muestra se compuso por docentes $(\mathrm{N}=66)$ y familiares de alumnos $(\mathrm{N}=22)$, sumando un total de 88 participantes. Utilizamos como herramienta el cuestionario: "Liderando la Educación Inclusiva" (LEI-Q). Se trata de una investigación de corte transversal ex post-facto con dos partes, una primera descriptiva y una segunda inferencial. En cuanto al análisis inferencial que planteamos, nos permitió comparar, por una parte, las diferencias de liderazgo inclusivo entre centros públicos y privados, y por otra, las perspectivas de los equipos docentes y las familias con respecto al mismo. Resultados: Los análisis de los datos obtenidos confirman, en primer lugar, la importancia que tiene el liderazgo inclusivo en los centros educativos, en segundo, que no existen diferencias significativas en el liderazgo inclusivo llevado a cabo en centros públicos y privados concertados y en tercero, la existencia de diferencias entre la perspectiva de los docentes y las familias. Conclusiones: Los resultados nos muestran que, en los centros estudiados, el Liderazgo Inclusivo está sustancialmente implantado.
\end{abstract}

Palabras clave: Liderazgo | Escuela Inclusiva

\section{Analysis of Inclusive Leadership in Primary and Secondary Schools in Granada}

Abstract: The fact that our society is increasingly heterogeneous makes it necessary to promote processes of social and educational inclusiveness. Acknowledging that school leaders are the key to promoting changes and that they play a significant role in the path to improvement, it makes sense to research inclusive leadership. Objective: This study aims to analyze inclusive leadership in both public and private educational centers located in the city of Granada. Method: this research study was carried out during the 2015/16 academic year. The sample was made up of teachers $(N=66)$ and relatives of students $(\mathrm{N}=22)$, with a total of 88 participants. The instrument used was the questionnaire: "Leading Inclusive Education" (LEI-Q). This is an ex post-facto cross-sectional investigation with two parts, the first part is descriptive and the second is inferential. In the inferential analysis proposed, a comparison was made between differences in inclusive leadership in the public and private institutions on the one hand and the perspectives of teaching teams and families on the other. Results: The analysis of the data obtained confirms the importance of inclusive leadership in schools and that there are differences between the perspectives of teachers and families, but no significant difference between public and private schools. Conclusions: The results show that Inclusive Leadership is substantially implemented at the centers studied.

Keyword: Leadership | Inclusive Schools

\section{Introducción}

Conseguir una educación de calidad en una sociedad cada vez más diversa y multicultural hace necesario propiciar procesos de inclusión (León 2012). De este modo, desde este artículo abordamos el liderazgo como forma de potenciar la inclusión en los centros educativos, pues existen evidencias de que éste es una pieza clave en el cambio y mejora de los mismos (Bolívar, López y Murillo, 2013). Como 
punto de apoyo para conseguir una auténtica justicia social e inclusión, Murillo y Hernández (2014) exponen cuatro premisas básicas. En su cuarta premisa señala que el líder escolar incide directamente con su comportamiento y actitudes a la consecución de una cultura inclusiva.

Para acercarnos a la esencia de la inclusión, hemos de concebirla como un proceso continuo de deconstrucción y reconstrucción de la cultura escolar, de las políticas y de las prácticas de cada centro, con el fin de descubrir barreras que frenen e imposibiliten el aprendizaje y la participación del alumnado (Booth y Ainscow, 2000; Ainscow, Booth y Dyson, 2006; León, 2011). De esta forma, no podemos entender la inclusión como una característica marginal o minoritaria del sistema educativo, sino como parte esencial para la construcción del sistema social deseado (Echeita, 2012). Por ello, Escudero y Martínez (2011) ponen en alza que "la educación inclusiva no es un enfoque inspirado en opciones caritativas y particulares, sino en imperativos morales y de justicia social" (p.88). De esta forma, Casanova (2011) afirma tajantemente que la educación, si no es inclusiva, no es educación.

En cuanto al liderazgo, es un término amplio y difícil de definir. Leithwood y Riehl (2009, p.18), consideran que el liderazgo: "existe dentro de relaciones sociales y sirve a fines sociales"; "implica un propósito y una dirección"; "es un proceso de influencia"; "es una función" y "es contextual y contingente". El liderazgo se puede ejercer en distintos ámbitos, pero al que nos referiremos en esta investigación es al liderazgo educativo, el cual se define como: "la labor de movilizar e influenciar a otros para articular y lograr las intenciones y metas compartidas de la escuela" (Leithwood y Riehl, 2009, p.20). Bolívar (2010) concreta que las metas a las que se debe aspirar deben ser propiamente pedagógicas. Como recoge este mismo autor, para constatar la gran importancia que tiene este liderazgo en el ámbito escolar, el informe Mackinsey (2007) y el informe TALIS de la OCDE (2008), lo sitúan como segundo factor más relevante, después de la práctica docente en el aula, para la consecución de logros de aprendizaje del alumnado. Hemos de puntualizar que la influencia que ejerce el liderazgo sobre el aprendizaje del alumnado es indirecta, transmitiéndose mediante una buena organización escolar y el fomento de la calidad en la enseñanza, viéndose potenciada de esta forma, además, la calidad del profesorado (Bolívar-Botía, 2010).

Con estos dos conceptos previos, surge el liderazgo inclusivo, el cual, siguiendo a Booth y Ainscow (2000) debe estar enfocado principalmente a conseguir una cultura inclusiva, la creación de una comunidad escolar en la que cada miembro es valorado y se fomente su nivel de logro y además se caracterice por ser acogedora, segura, colaboradora y estimulante. Pretende en última instancia desarrollar valores inclusivos compartidos para toda la comunidad educativa en general, alumnado, profesorado y familias principalmente. Con el fin de arrojar luz al asunto, diversos autores han definido el liderazgo inclusivo. Entre ellos, por una parte, Essomba (2006), lo define como aquel liderazgo en el que el líder debe enfocar su centro de actuación a las relaciones humanas, por encima de estrategias y recursos. Además, ha de concebir la comunidad educativa como un sistema de relaciones abierto, y encaminar el cambio y la transformación hacia el entorno social. Por otra parte, Ryan (2006) sostiene que el liderazgo inclusivo consiste en una serie de prácticas distintas como: educar a los participantes, desarrollar conciencia crítica, alimentar el diálogo, haciendo hincapié en la práctica de aula y aprendizaje de estudiantes, adoptar decisiones inclusivas y formulación de políticas estratégicas e incorporando enfoques de toda la escuela. Ambas definiciones no son excluyentes sino complementarias. Concluye este mismo autor que una dirección que priorice el rendimiento académico ante el desarrollo integral de las personas, contribuirá al fomento de acciones y políticas de competitividad y exclusión. 
A pesar de los esfuerzos por aportar una definición concreta, resulta muy difícil llegar a definir un liderazgo inclusivo generalizable, pues diversos estudios sobre el campo, coinciden en que no hay unas prácticas fijas, ni un tipo de liderazgo particular que sea clave para llevar acabo un liderazgo inclusivo, sino que se necesita una predisposición y agilidad para cambiar de un estilo de liderazgo a otro y elegir las estrategias más afines de cada uno, para resolver los problemas particulares de cada centro (Fernández-Batanero y Hernández, 2013). Aun sabiendo esto, tras un estudio en 13 escuelas de Chipre, Angelides, Antoniou y Charalambus (2010) concluyen que en esas escuelas se dan cinco condiciones clave para conseguir metas generalizables que apuntan a una escuela inclusiva. 1) Implicación del profesorado en ciertas actividades relacionadas con la educación inclusiva, 2) Co-enseñanza entre el profesorado y el profesor de educación especial; 3) Promoción de una cultura colaborativa con el fin de cubrir las necesidades de todo el alumnado; 4) Dar igualdad de oportunidades a todos los niños con amor, cuidado y aceptación; 5) La implicación de la comunidad escolar y las familias.

Con estas premisas, mediante este estudio, pretendemos conocer el grado de liderazgo inclusivo que desempeñan los equipos directivos en centros educativos de Granada; identificar cuáles son los déficits y fortalezas más habituales con respecto al Liderazgo Inclusivo en los centros estudiados; comprobar si existen diferencias en el desempeño de Liderazgo Inclusivo entre centros públicos y privados. $Y$ entre la percepción de equipos docentes y familias en las dos primeras dimensiones del cuestionario sobre Liderazgo Inclusivo.

\section{Método}

En la presente investigación empleamos una metodología cuantitativa, basada en un cuestionario. Concretamente se trata de una investigación ex post-facto (Bisquerra, 2014) donde se ha llevado a cabo un análisis descriptivo con el que se da una visión general de la situación de cinco centros educativos de Granada referente al liderazgo inclusivo y un análisis inferencial con el que se extraen las diferencias existentes entre los grupos de comparación.

\section{Participantes}

En esta investigación participan un total de 5 centros, de los cuales, dos son públicos y tres privados-concertados. Es preciso apuntar que tenemos autorización para su estudio y acceso por parte de la Consejería de Educación de la Junta de Andalucía. De los 88 sujetos participantes, 34 son hombres (37\%) de los cuales 3 pertenecientes al grupo de familias (padres) y 28 pertenecen al equipo docente; y 55 son mujeres $(63 \%)$ de las cuales 18 pertenecientes al grupo de familias (madres) y 37 pertenecen al equipo docente. En la tabla 1, especificamos las características de los sujetos participantes en la investigación. Aclaramos que "N.E.D." se refiere al número de participantes de equipos docentes y "N.Fam." al número de sujetos participantes pertenecientes a familias.

Tabla 1. Características de los centros y sujetos participantes

\begin{tabular}{lllcccc}
\hline Centros & Niveles & Carácter & N.E.D & N. Fam. & N.T & N.T. priv. y publi. \\
\hline Centro 1 (C1) & Primaria/Secundaria & Privado & 13 & 0 & 13 & \\
\cline { 1 - 5 } Centro 2 (C2) & Primaria & Privado & 12 & 0 & 12 & \\
\cline { 1 - 5 } Centro 3 (C3) & Primaria/Secundaria & Privado & 11 & 6 & 17 & $\mathbf{4 2}$ \\
\hline Centro 4 (C4) & Primaria & Público & 10 & 10 & 20 & \multirow{2}{*}{} \\
\cline { 1 - 5 } Centro 5 (C5) & Secundaria & Publico & 20 & 6 & 26 & $\mathbf{4 6}$ \\
\hline
\end{tabular}




\section{Instrumento}

El instrumento que se ha utilizado es el "Cuestionario: Liderando la Educación Inclusiva" (LEI-Q) (León, et al 2016). Es un cuestionario de tipo Likert con 4 opciones de respuesta (1. aún no implantado; 2. parcialmente implantado; 3. sustancialmente implantado; 4. totalmente implantado), y está compuesto por setenta ítems divididos en cuatro dimensiones: I. Apertura a la comunidad (comprueba si el equipo directivo lleva a cabo iniciativas y acciones que potencia la apertura del centro hacia el entorno y las familias) II. El centro como comunidad inclusiva (recoge iniciativas destinadas a generar visiones compartidas, objetivos grupales, altas expectativas grupales, generar debates, ...) III. Comunidad profesional de aprendizaje (aúna acciones dirigidas a promover la formación y desarrollo profesional del profesorado creando comunidades profesionales de aprendizaje) IV. Gestionar procesos de enseñanza-aprendizaje (recoge iniciativas y acciones para promover mejoras y favorecer la coordinación en el proceso de enseñanza aprendizaje).

Se trata de un cuestionario validado por juicio de expertos y de jueces en relación a cuatro indicadores: coherencia, claridad, relevancia y pertinencia. Con respecto a la fiabilidad, el Alpha de Cronbach para el cuestionario completo es de ,989, y por dimensiones se han obtenido valores que van desde ,968 (Dimensión IV) y ,925 (Dimensión I), con un nivel de confianza del 95\% $(\mathrm{p}<, 05)$. En cuanto a la validez, los cocientes de correlación de Spearman oscilan entre ,962 (Dimensión III) y ,939 (Dimensión I). Por tanto, es un cuestionario altamente fiable y válido.

Tabla 2. Fiabilidad y validez de constructo del instrumento

\begin{tabular}{lccc}
\multicolumn{1}{c}{$\begin{array}{c}\text { Total del cuestionario } \\
\text { Alpha= '989 }\end{array}$} & $\begin{array}{c}\text { N de } \\
\text { elementos }\end{array}$ & $\begin{array}{c}\text { Fiabilidad de los } \\
\text { bloques } \\
\text { (Alpha de } \\
\text { Cronbach) }\end{array}$ & $\begin{array}{c}\text { Promedio correlación entre } \\
\text { criterios (Spearman) }\end{array}$ \\
\hline $\begin{array}{l}\text { Dimensión I. Apertura hacia la } \\
\text { comunidad }\end{array}$ & 15 & .925 & .939 \\
$\begin{array}{l}\text { Dimensión II. El centro como } \\
\text { comunidad inclusiva }\end{array}$ & 22 & .958 & .959 \\
$\begin{array}{l}\text { Dimensión III. Comunidad profesional } \\
\text { de aprendizaje }\end{array}$ & 11 & .940 & .962 \\
$\begin{array}{l}\text { Dimensión IV. Gestionar procesos de } \\
\text { enseñanza-aprendizaje. }\end{array}$ & 22 & .968 & .948 \\
\hline
\end{tabular}

La correspondencia entre dimensiones e ítems del cuestionario se representa en la siguiente tabla.

Tabla 3. Correspondencia entre dimensiones e ítems del cuestionario

\begin{tabular}{lcc}
\hline Dimensiones & Ítems & $\mathbf{N}$ \\
\hline I. Apertura hacia la comunidad & $1-15$ & 15 \\
\hline II. El centro como comunidad inclusiva & $16-37$ & 22 \\
\hline III. Comunidad profesional de aprendizaje & $38-48$ & 11 \\
\hline IV. Gestionar procesos de enseñanza-aprendizaje & $49-70$ & 22 \\
\hline
\end{tabular}

Pretendemos con este cuestionario, no solo obtener información para la investigación, sino también, dar a los centros una herramienta de autoevaluación, que les permitan detectar sus fortalezas y debilidades para establecer, en última instancia, planes de mejora enfocados a modelos de buenas prácticas. 


\section{Procedimiento}

El procedimiento que se ha seguido es el siguiente: Planteado el problema a investigar, iniciamos la revisión bibliográfica, tras la cual, se procedió a la divulgación del cuestionario, la cual se ha realizado de forma personal, visitando cada centro tras ponernos en contacto con el equipo directivo previamente. Una vez cumplimentados y recogidos, pasamos al volcado de los datos en el programa SPSS 22.0. y con los datos organizados, iniciamos el análisis descriptivo e inferencial. Tras estos análisis, y con el objetivo de profundizar más en uno de los temas, se ha llevado a cabo un análisis descriptivo e inferencial, comparando ítem por ítem. Por último, discutimos los resultados obtenidos

\section{Análisis de los datos}

Para el análisis de los datos obtenidos, en primer lugar, se ha realizado un análisis descriptivo, con el objetivo de conocer de forma global la situación del liderazgo inclusivo percibido por los equipos docentes y las familias en los centros de educación primaria y secundaria de Granada, además, de revelar debilidades y fortalezas referentes a las cuatro dimensiones del cuestionario. Las familias han contestado las dos primeras dimensiones, y los equipos docentes las cuatro, puesto que las dos últimas están centradas específicamente en aspectos del centro. Con este análisis, redactamos un informe para cada centro participante en la investigación, con la intención de aportar el feedback correspondiente a los centros, y que puedan dirigir sus esfuerzos a mejorar sus debilidades y a seguir trabajando en sus fortalezas. En este informe se les comunica el grado de Liderazgo Inclusivo que han obtenido en su centro en valores porcentuales. Después, se les presenta pormenorizadamente el resultado obtenido en cada uno de los ítems mediante gráficas de cada dimensión. Además, se señala el porcentaje obtenido de cada dimensión, y los tres ítems mejor y peor valorados, para que el centro pueda sacar sus propias conclusiones. Por último, se le hace una consideración final, y se les facilita un código $Q R$, de acceso al cuestionario para que puedan autoevaluarse en cualquier momento.

En segundo lugar, se realizó un análisis inferencial, con el objeto de estudiar la existencia de posibles diferencias en función de las siguientes variables de identificación: la titularidad de los centros (públicos y privados-concertados), para todo el cuestionario, y la identidad (familia y equipo docente), para las dos primeras dimensiones del cuestionario. Se ha llevado a cabo la prueba estadística U de MannWhitney para muestras no paramétricas.

En último lugar, y tras haber encontrado diferencias estadísticamente significativas entre la perspectiva de liderazgo inclusivo de los equipos docentes y las familias, llevamos a cabo un análisis descriptivo e inferencial centrándonos en las dos primeras dimensiones concretamente.

\section{Resultados}

De forma global, para los centros estudiados, se obtiene una media de 3,2 puntos y una desviación típica de 0,74. En la gráfica 1 mostramos el grado de Liderazgo Inclusivo obtenido por cada uno de los centros educativos que han participado en el estudio. 


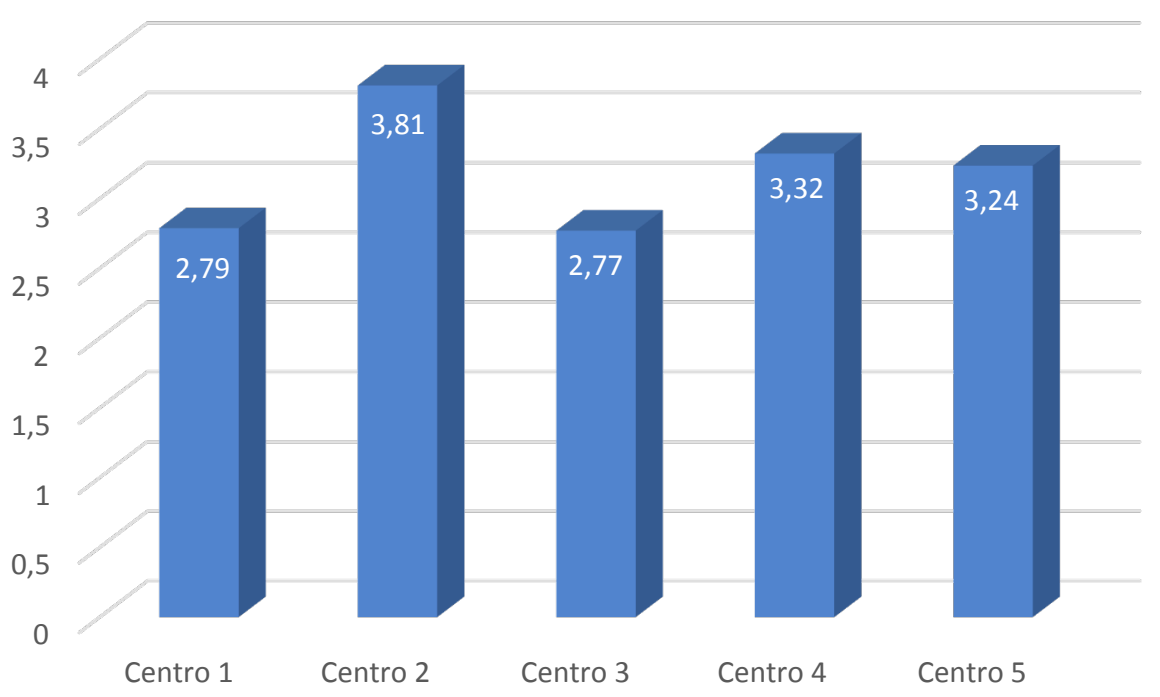

Gráfica 1: Grado de Liderazgo Inclusivo en centros educativos de primaria y secundaria de Granada

Los valores obtenidos por los mismos oscilan entre 3,81 (C2), para el centro con mayor grado de Liderazgo Inclusivo, y 2,77 (C3), para el centro que ha obtenido un resultado global más bajo. Hemos de destacar que, en el cómputo global, obtiene más grado de LI los centros públicos (C4 y C5) con una media de 3,26, frente al 3,13 de los privados ( $1, C 2$ y C3), aunque el valor más alto lo ha conseguido un privado (C2). En cuanto a la desviación típica, oscila entre 0,20 (C2) y 1,14 (C1).

En la siguiente tabla mostramos las dimensiones que prioriza cada uno de los centros estudiados, incluyendo las respuestas de las familias.

Tabla 4. Resumen de puntuaciones por centro y dimensión

\begin{tabular}{lcccccc}
\hline & C1 & C2 & C3 & C4 & C5 & Total \\
\hline Dimensión 1 & 2,759 & 3,773 & 2,733 & $\mathbf{2 , 9 7 7}$ & 3,031 & $\mathbf{3 , 0 2 3}$ \\
Dimensión 2 & 2,860 & 3,782 & 2,711 & 3,164 & 3,208 & 3,130 \\
Dimensión 3 & $\mathbf{2 , 5 8 7}$ & $\mathbf{3 , 7 2 7}$ & $\mathbf{2 , 6 6 9}$ & 3,291 & 3,100 & 3,079 \\
Dimensión 4 & 2,948 & 3,959 & 2,983 & 3,459 & 3,366 & 3,349 \\
\hline
\end{tabular}

De acuerdo con estos resultados, podemos comprobar que todos los centros participantes obtienen la mayor puntuación en la dimensión 4, "Gestionar procesos de enseñanza-aprendizaje", mientras que la menos valorada es la relativa a la "Comunidad profesional de aprendizaje", siendo esta también la menos valorada por los centros privados ( $1, \mathrm{C}^{2}$ y C3) y la 1 "Apertura hacia la comunidad", por los centros públicos (C4 y $\mathrm{C} 5$ ).

En cuanto al análisis inferencial, realizamos la prueba no paramétrica $U$ de MannWhitney, para las variables titularidad del centro en primer lugar, y en segundo lugar para la identidad.

En relación a la titularidad del centro no se encuentran diferencias significativas $(p \leq$ 0,05 ) entre centros públicos y privados, por tanto, no profundizaremos más en este aspecto. 
Tabla 5. Contraste de diferencias en la opinión de Centros Privados y Centros Públicos para las dimensiones 1, 2, 3 y 4

\begin{tabular}{lcccc}
\hline & Dimensión 1 & Dimensión 2 & Dimensión 3 & Dimensión 4 \\
\hline U de Mann-Whitney & 866,000 & 893,500 & 537,500 & 474,500 \\
\hline Sig. Asintónica & .403 & .544 & .974 & .398 \\
\hline
\end{tabular}

Sin embargo, para la variable identidad, considerando como grupos de comparación los equipos docentes y las familias, (en las dimensiones 1 y 2), obtenemos que existen diferencias estadísticamente significativas $(p \leq 0,05)$ en ambas dimensiones. Los datos se recogen en la siguiente tabla.

Tabla 6. Contraste de diferencias en la opinión de Equipos Docentes y Familias para las dimensiones 1 y 2

\begin{tabular}{ccc}
\hline & Dimensión 1 & Dimensión 2 \\
\hline U de Mann-Whitney & 430,500 & 435,500 \\
\hline Sig. Asintónica & .004 & .005 \\
\hline
\end{tabular}

Tras este hallazgo, profundizamos en estas dos dimensiones, para conocer concretamente, en qué ítems difieren ambos grupos. Para ello llevamos a cabo un análisis descriptivo y otro inferencial.

En primer lugar, de acuerdo con los resultados del análisis descriptivo, de la Dimensión I: "apertura hacia la comunidad" podemos concluir que los ítems 8 (ofrecimiento de instalaciones y recursos) y 13 (atención a demandas y necesidades de las familias) se encuentran entre los mejor valorados tanto por los equipos docentes como por las familias. El ítem 3 (relación escuela-entorno laboral) se encuentra entre los peor valorados en ambos grupos. El ítem 5 (propuesta de actividades educativas fuera del centro), se encuentra entre los mejor valorados por los equipos docentes, sin embargo, es uno de los peor valorados por las familias. De forma global, los equipos directivos valoran más positivamente que las familias la apertura hacia la comunidad, con una diferencia de 0,32 puntos. Por último, tras realizar la $U$ de Mann-Whitney para los ítems de esta dimensión, obtenemos que hay diferencias significativas $(p \leq 0,05)$ en los ítems referentes a: promoción de la colaboración con el mundo empresarial (ítem 3); propuesta de actividades educativas fuera del centro (ítem 5); participación en acciones formativas emprendidas por instituciones/organizaciones de la comunidad (ítem 7); puesta en conocimiento de las familias de la propuesta curricular que orienta la acción educativa del centro (ítem 9); promoción de acciones que facilitan la comunicación y participación de todas las familias en las actividades educativas dentro y fuera del centro (ítem 11); previsión de medidas para contrarrestar la influencia negativa que pudiera tener la situación familiar en el éxito de sus estudiantes (ítem 15).

En segundo lugar, por lo que respecta a la Dimensión II "el centro como comunidad inclusiva": del análisis descriptivo concluimos que el ítem 23 (proceso de admisión y matriculación) aparece entre los más valorados tanto para los equipos docentes como para las familias, y el ítem 16 (recogida de necesidades del profesorado, alumnado y resto del personal del centro) aparece en los menos valorados, tanto para los equipos docentes como para las familias. Y en general, los equipos directivos valoran más positivamente que las familias el centro como comunidad inclusiva, con una diferencia de 0,34 puntos. En cuanto al análisis inferencial, tras realizar la $U$ de Mann-Whitney para los ítems de esta dimensión, obtenemos que hay diferencias significativas ( $p \leq$ $0,05)$ en los ítems referentes a: disposición de un procedimiento de recogida de información sobre necesidades del profesorado, alumnado y resto del personal del centro, (ítem 16); preocupación de que los servicios que ofrece el centro respeten las 
diferentes necesidades del alumnado (ítem 17); preocupación por dotar al centro de recursos materiales y humanos para promover procesos de mejora (ítem 19); promoción entre el profesorado una visión compartida sobre la organización, metas y actividades para hacerles partícipes de un proyecto común (ítem 21); desarrollo de programas educativos para prevenir actitudes discriminatorias entre el alumnado (ítem 26); generación de oportunidades para que todos los miembros de la comunidad educativa participen de forma efectiva en las decisiones (ítem 28); disposición de mecanismos para documentar los cambios y mejoras emprendidas (ítem 30); preocupación por garantizar el acceso a las instalaciones y servicios del centro a todo el alumnado (ítem 37).

\section{Discusión}

De manera general, hemos comprobado que el liderazgo inclusivo que ejercen los equipos directivos de los centros estudiados es valorado muy positivamente, al igual que los directores de centros educativos de Ceuta (Cuevas, Díaz e Hidalgo, 2008). Por tanto, como estos autores, podemos concluir que estamos en una posición privilegiada para llevar a cabo y dinamizar procesos de cambio que tienden a mejorar la escuela en su globalidad.

De las cuatro categorías que caracterizan un liderazgo exitoso, según Leithwood (2004), la cuarta hace referencia a la gestión de procesos de enseñanza-aprendizaje, la cual ha sido una de las dimensiones más valoradas en nuestro estudio, al igual que en el de León, et al. (2016). Por tanto, podemos decir según los resultados obtenidos, que en el total de los centros estudiados se apunta hacia un liderazgo exitoso. Por otro lado, al aparecer como menos valorada la "apertura hacia la comunidad" deducimos que los equipos directivos deben llevar a cabo más iniciativas que potencien la apertura del centro hacia el entorno (asociaciones vecinales, ONG, empresas, servicios sociales, etc.) y las familias.

A pesar de haber obtenido diferencias significativas generales entre equipos docentes y familias, en 14 de 37 ítem contestados por ambos colectivos, hay puntos de encuentro entre ambos grupos. En primer lugar, en cuanto a la "apertura hacia la comunidad" (Dimensión 1), coinciden en considerar como aspecto mejor valorado la atención desde el centro a la comunidad. Además, ambos grupos, coinciden en considerar como peor valorado la colaboración con el mundo empresarial.

La diferencia más acusada, es la relativa a proponer actividades educativas fuera del centro que, en el caso de los equipos docentes, se encuentra entre los ítems mejor valorados, y al contrario en las familias. En este punto, cabe preguntarse, ¿están las familias informadas de todas las actividades educativas fuera del centro?, ¿se proponen muchas, pocas o las oportunas actividades educativas fuera del centro? Quizás este tema sea una puerta abierta para una futura investigación.

En definitiva, la línea que marca las diferencias significativas con respecto a la apertura hacia la comunidad (Dimensión 1), está enfocada a la falta de comunicación entre el centro y las familias, en ambas direcciones, y precisamente esta doble vía de información es clave para conseguir el éxito escolar del alumnado (Garreta, 2015). El centro debería ofrecer más información a las familias con respecto a aspectos curriculares que orientan la acción educativa del centro, para que de esta forma la familia pueda trabajar desde su campo de actuación con sus hijos/as en la misma línea de trabajo que el centro y además pueda ofrecer propuestas de mejora. En la otra vertiente, el centro debe conocer más a las familias para que desde el centro se 
pueda contrarrestar posibles influencias negativas que pueda tener desde el entorno familiar.

En segundo lugar, en cuanto al "centro como comunidad inclusiva" (Dimensión 2), existen coincidencias tanto para el conjunto de los aspectos mejor valorados, (concretamente la admisión y matriculación), como para el conjunto de los peor valorados, (específicamente relativo al conocimiento de las necesidades del profesorado, alumnado y personal del centro). En este sentido, Hines (2008) destaca que para que el equipo directivo pueda ejercer bien su labor como mediador de conflictos, es esencial que se propicie un ambiente en el que docentes, alumnos y comunidad pueda expresarse con comodidad. Es decir, llevar a cabo medidas y estrategias comunicativas esenciales para poder conocer $\mathrm{y}$, consecuentemente, atender las necesidades de la comunidad educativa en general. Por tanto, para avanzar hacia un liderazgo inclusivo, éste es otro de los puntos que los centros participantes deben mejorar. De las diferencias significativas entre docentes y familias de esta dimensión surgen propuestas de mejora. De manera general, los centros deberían generar mecanismos para descubrir necesidades del profesorado, alumnado y personal del centro, prevenir actitudes discriminatorias y para documentar cambios y mejoras y fomentar la incorporación de las familias en la medida de lo posible en la vida del centro para conseguir un proyecto común y una participación efectiva en las decisiones. De esta forma, estaríamos dotando al centro de recursos humanos para promover procesos de mejora.

Con respecto a la última conclusión, los centros públicos coinciden con la media global, al considerar como menos valorada la "apertura hacia la comunidad" (dimensión 1). En este sentido, Murillo y Hernández (2014) afirman que no debemos olvidar la importancia de sostener estrechas relaciones con miembros de la comunidad en la que se enmarca la escuela, invitando a participar en actividades del centro a personas externas, y ofreciendo espacios donde puedan trabajar fuera del edificio escolar, por tanto, es importante que este aspecto mejore.

En cuanto a los centros privados, los participantes del estudio consideran que la "comunidad profesional de aprendizaje" (dimensión 3) es la más deficitaria, y, por tanto, aquella sobre la que hay que insistir en el plan de mejora de forma más inmediata, ya que, si se quiere conseguir un liderazgo inclusivo y, por ende, una escuela inclusiva, se deben crear comunidades profesionales de aprendizaje (León, 2012) por las ventajas educativas que implican (Ryan, 2006). Entre estas, la priorización del aprendizaje para todos los miembros de la comunidad, el cuidado de las relaciones entre los mismos, difundir conocimiento y habilidades, proporcionar estrategias de autorrealización, (Collinson, Cook y Conley, 2006), aumento del compromiso con los resultados, mejora de forma continua, promoción de una cultura de colaboración y de un liderazgo compartido y apoyo (NAESP, 2008).

Por último, en el cómputo global, los centros públicos obtienen más grado de liderazgo inclusivo que los centros privados, pero, a pesar de estas diferencias, los resultados en las pruebas, apuntan que no hay diferencias significativas entre centros públicos y privados, a diferencia de los hallazgos de Cuevas, Díaz e Hidalgo (2008), en su estudio sobre liderazgo de los directores, que sí encontraron diferencias significativas a favor de los directivos de centros privados. 


\section{Referencias}

Ainscow, M., Both, T., y Dyson, A. (2006). Inclusion and the standards agenda: negotiating policy pressures in England. International journal of inclusive education, 10(4-5), 295-308.

Angelides, P., Antoniou, E., y Charalambous, C. (2010). Making sense of inclusion for leadership and schooling: a case study from Cyprus. International journal of leadership in education, 13(3), 319-334

Bisquerra, R. (Coord.) (2014). Metodología de la investigación educativa (4a ed.). Madrid: La Muralla S.A.

Bolívar, A. (2010). El liderazgo educativo y su papel en la mejora: una revisión actual de sus posibilidades y limitaciones. Psicoperspectivas, 9(2), 9-33.

Bolívar, A. (2010). ¿Cómo un liderazgo pedagógico y distribuido mejora los logros académicos? Revisión de la investigación y propuesta. MAGIS, 3(5), 79-106.

Bolívar, A., López, J. y Murillo, F.J. (2013). Liderazgo en las instituciones educativas. Una revisión de líneas de investigación. Revista Fuentes, 14, 15-60.

Booth, T. y Ainscow, M. (2000). Guía para la evaluación y la mejora de la educación inclusiva. Desarrollando el aprendizaje y la participación en las escuelas. Madrid. Universidad autónoma de Madrid.

Casanova, M. (2011). Educación inclusiva. Un modelo de futuro. Madrid: Wolters Kluve.

Collinson, V., Cook, T., y Conley, S. (2006). Organizational learning in schools and school Systems: Improving learning, teaching and leading. Theory into Practice, 45(2), 107-116.

Cuevas, L., Díaz, R., e Hidalgo, H. (2008). Liderazgo de los directores y calidad de la educación. Un estudio del perfil de los directivos en un contexto pluricultural. Profesorado, revista de currículum y formación del profesorado, 12(2), 1-20.

Echeita, G. (2012). Competencias esenciales en la formación inicial de un profesorado inclusivo. Un proyecto de la agencia europea para el desarrollo de las necesidades educativas especiales. Tendencias pedagógicas, 19, 7-24.

Escudero, J., y Martínez, B. (2011). Educación inclusiva y cambio escolar. Revista Iberoamericana de Educación, 55, 85-105.

Essomba, M. (2006). Liderar escuelas interculturales e inclusivas. Equipos directivos y profesorado ante la diversidad cultural y la inmigración, Barcelona, España: Graó.

Fernández-Batanero, J. M., y Hernández, A. (2013). Liderazgo directivo e inclusión educativa. Estudio de casos. Perfiles educativos, 35(142), 27-41.

Garreta, J. (2015). La comunicación familia-escuela en educación infantil y primaria. Revista de la asociación de sociología de la educación (RASE), 8(1), 71-85.

Leithwood, K., y Riehl, C. (2009). ¿Qué sabemos sobre liderazgo educativo? En K. Leithwood. (Ed.), ¿Cómo liderar nuestras escuelas? Aportes desde la investigación (pp. 17-33). Santiago de Chile, Chile: Salesianos.
Leithwood, K., Seashore Louis, K., Anderson, S., y Wahlstrom, K. (2004). How leadership influences student learning. Recuperado de www.wallacefundation.org

León, MJ. (2011). La situación de la formación en educación inclusiva en los nuevos títulos de grado de maestro en España. Revista interuniversitaria de formación del profesorado, 70, 145-163.

León, MJ. (2012). El liderazgo para y en la escuela inclusiva. Educatio Siglo XXI, 30(1), 133-160.

León, MJ., López, MC., Romero, A., Crisol, E., Hinojosa, E., y Moreno, R. (noviembre de 2016). Liderando la educación inclusiva en centros de educación primaria y secundaria. En $\mathrm{JL}$. Bernal (Coordinador), XIV Congreso interuniversitario de organización de instituciones educativas (CIOIE). Liderazgo inclusivo y para la justicia social. Congreso llevado a cabo en Zaragoza, España.

Murillo, F.J., y Hernández, R. (2014). Liderando escuelas justas para la justicia social. Revista internacional de educación para la justicia social, 3(2), 13-32.

Ryan, J. (2006). Inclusive leadership and social justice for schools. Leadership and policy in schools, 5(1), 3-17. 